\title{
Estudo da influência do condicionamento higrotérmico nas propriedades viscoelásticas de compósitos termoplásticos
}

\section{Study of the influence on higrothermal conditioning on viscoelastic properties of thermoplastic composites}

\author{
Maria Cândida Magalhães de Faria', Pedro Carlos de Oliveira², Bruno Ribeiro', \\ José Maria Fernandes Martet ${ }^{3}$ e Edson Cocchieri Botelho ${ }^{1 *}$ \\ 'Departamento de Materiais e Tecnologia, Universidade Estadual Paulista “Júlio de Mesquita Filho" - \\ UNESP, Guaratinguetá, SP, Brasil \\ ${ }^{2}$ Departamento de Engenharia Química, Universidade de São Paulo - USP, Lorena, SP, Brasil \\ ${ }^{3}$ Empresa Brasileira de Aeronáutica - EMBRAER, São José dos Campos, SP, Brasil \\ *ebotelho@pq.cnpq.br
}

\begin{abstract}
Resumo
O contínuo crescimento na utilização de compósitos termoplásticos em componentes estruturais na indústria aeroespacial deve-se, primordialmente, à flexibilidade de projeto, excelência de suas propriedades mecânicas e baixa massa específica, aliadas aos elevados valores de resistência mecânica e rigidez e baixa incidência de corrosão. No entanto, componentes que requerem exigências estruturais, quando expostos a ambientes agressivos como elevada temperatura e umidade, podem ter suas propriedades mecânicas sensibilizadas por esses fatores ambientais, e devem ser cuidadosamente avaliados antes de serem colocados em serviço. Embora existam diversos trabalhos na literatura reportando este problema para compósitos estruturais, poucos estudos relacionam, de maneira sistemática, a avaliação da absorção de umidade com o comportamento viscoelástico de matrizes de PPS reforçadas com fibras de carbono, quando este compósito é utilizado em aplicações aeronáuticas. Sendo assim, o objetivo deste trabalho é avaliar a influência do condicionamento higrotérmico nas propriedades viscoelásticas de laminados PPS/fibras de carbono. Os resultados mostram que os laminados condicionados em câmara higrotérmica apresentaram absorção de umidade segundo as leis de Fick, porém, não foram observadas variações significativas na temperatura de transição vítrea quando comparado compósitos condicionados e não condicionados.
\end{abstract}

Palavras-chave: compósitos termoplásticos, efeito higrotérmico, propriedades mecânicas.

\begin{abstract}
Continued growth in the use of thermoplastic composites for structural components in the aerospace industry due, primarily, to the design flexibility, excellent mechanical properties and low density, combined with the high values of strength and stiffness and low incidence of corrosion meeting the stringent performance requirements of these structures while in service. However, components that requires structural requirements when exposed to harsh environments such as high temperature and moisture, can have their mechanical properties reduced by these environmental factors, and should be carefully evaluated before being placed in service. Although there are several studies in the literature reporting this problem for structural composites, few studies relate in a systematic way, assessing the moisture absorption with the viscoelastic behavior of PPS matrix reinforced with carbon fibers, when the composite is used in aeronautical applications. Thus, the aim of this study is to evaluate the influence of hygrothermal conditioning on the viscoelastic properties of PPS/carbon fiber composites. The results show that the laminates conditioned in a hygrothermal chamber had moisture absorption according to Fick laws, but it was not observed a significant glass transition temperature variation when compared conditioned and not conditioned specimens.
\end{abstract}

Keywords: thermoplastic composites, hygrothermal effect, mechanical properties.

\section{Introdução}

A indústria aeroespacial foi a pioneira no desenvolvimento tecnológico de materiais compósitos utilizados em projetos de aeronaves, mísseis, satélites e foguetes por necessitarem de componentes de baixa densidade e que atendessem aos severos requisitos de resistência mecânica em serviço ${ }^{[1,2]}$. Devido às suas características e desempenho os compósitos tornaram-se uma classe atrativa de material como substituintes das tradicionais ligas metálicas em aplicações 
aeroespaciais, levando a uma redução de peso do veículo com um consequente ganho em termos de economia de combustível, de extensão da autonomia de voo e de aumento de produtividade, possibilitando uma redução no custo final dos componentes na ordem de até $25 \% \%^{[3,4]}$.

A competição da indústria aeronáutica e os rígidos padrões de qualidade, visando maiores valores de resistência mecânica, química e térmica com produtos mais leves tem impulsionado novos desenvolvimentos científicos neste setor. Os compósitos poliméricos reforçados com fibras de carbono (CRFC) estão sendo amplamente empregados na indústria aeroespacial, e atualmente sua utilização vai desde simples painéis dos interiores das aeronaves até estruturas primárias e secundárias das fuselagens, empenagens verticais e horizontais, e também dos painéis de comandos primários de $\operatorname{voo}^{[5,6]}$.

A maioria dos compósitos utilizados atualmente é constituída de matrizes termorrígidas que apresentam problemas de tensões em virtude do processamento e da natureza quebradiça da resina, falta de resistência à erosão e, quando aquecidos, podem apresentar mudanças químicas e estruturais limitando sua aplicação em algumas regiões de maior temperatura de serviço e não podem ser reciclados devido à presença de ligações cruzadas. Entretanto, os polímeros termoplásticos reforçados com fibras contínuas vêm se mostrando importantes substitutos aos termorrígidos e conquistando uma grande versatilidade de aplicações ${ }^{[6,7]}$.

Atualmente, muita atenção vem sendo dada à utilização de compósitos termoplásticos para aplicações estruturais, podendo ser citados o PEI (poli(éter-imida)), o PEKK (poli(éter-cetona-cetona)), o PEEK (poli(éter-éter-cetona)) e o PPS (poli(sulfeto de fenileno)), todos reforçados com fibras de vidro ou carbono ${ }^{[7,8]}$.

As aeronaves operam em condições ambientais diversificadas tais como: ar frio e seco ou quente e úmido, elevada ou baixa taxa de radiação ultravioleta, etc. Portanto, é importante que os materiais utilizados na construção dessas aeronaves mantenham suas propriedades durante todo o seu período de vida útil, mesmo nos ambientes mais severos. As propriedades mecânicas e físico-químicas dos compósitos de matriz polimérica podem ser degradadas por uma variedade de efeitos físicos e químicos; portanto, é importante entender tanto o material quanto o ambiente operacional a que este se encontra exposto. Efeitos tais como umidade, temperatura, radiação UV, dentre outros, presentes na atmosfera devem ser sempre considerados nos projetos de laminados estruturais ${ }^{[9-14]}$.

A umidade, por exemplo, penetra na matriz polimérica pelo processo de difusão até que a concentração de equilíbrio seja atingida, sendo esse mecanismo acentuado pelo aumento da temperatura. Neste caso, a umidade pode causar a expansão volumétrica da matriz polimérica, gerando pequenas variações dimensionais e tensões internas. A taxa de absorção de umidade no compósito pode ser aumentada por picos térmicos acima de $140{ }^{\circ} \mathrm{C}$, durante pequenos intervalos de tempo (típicos em voos supersônicos), que favorecem o surgimento de danos na forma de trincas superficiais no laminado, microtrincas na matriz e/ou na interface fibra/resina. Como resultado, a resistência à tração, rigidez e a resistência ao cisalhamento interlaminar dos laminados geralmente são reduzidas ${ }^{[15-20]}$.
Para os compósitos com matrizes poliméricas, a matriz do compósito absorve a umidade do ar, principalmente a elevadas temperaturas, onde o processo de difusão é intensificado. A polaridade da matriz tem grande influência na absorção de umidade pelo compósito. Quanto maior for a polaridade da matriz maior será a sua capacidade de absorção de água. Além das interações polares entre moléculas de água e a matriz, a configuração espacial da molécula do polímero utilizada como matriz polimérica também influencia no processo de absorção de umidade pelo material.

Os efeitos ambientais causados pela temperatura e umidade relativa do ar podem ser reversíveis quando o período de exposição é de curta duração. Porém, quando a exposição ocorre em ciclos prolongados, onde a combinação da umidade com mudança de temperatura está presente, os efeitos produzidos são irreversíveis, devido à afinidade da água por grupos funcionais específicos de matrizes poliméricas de natureza polar. Nesse caso, geralmente ocorrem alterações destrutivas na interface reforço/matriz polimérica, devido à degradação das prováveis interações físico-químicas existentes entre a resina e a fibra. Como consequência desse fato, ocorre o descolamento da fibra, provocando a delaminação do compósito com a consequente redução das propriedades mecânicas e termofísicas do material compósito ${ }^{[14-22]}$.

Um dos efeitos mais acentuados da absorção de umidade em um compósito polimérico é a plasticização da matriz polimérica, levando geralmente a uma redução da temperatura de transição vítrea $\left(\mathrm{T}_{\mathrm{g}}\right)$ da matriz e mudanças nas propriedades mecânicas, tais como o módulo de elasticidade. A temperatura de transição vítrea é uma propriedade muito importante do material, pois define a temperatura de utilização para um determinado componente. Como esperado, a degradação nas propriedades da matriz polimérica afeta, principalmente, as propriedades do compósito dominadas pela matriz a elevadas temperaturas ${ }^{[23-25]}$.

Especificamente em se tratando da matriz PPS reforçada com fibras de carbono, poucos trabalhos vêm sendo reportados na literatura científica especializada envolvendo um estudo sistemático relacionando o ganho de umidade e eventuais mudanças no comportamento viscoelático, principalmente com o intuito de avaliar o modelo mais apropriado da absorção de umidade e difusividade da água para este compósito. Alguns trabalhos foram reportados anteriormente ${ }^{[26-28]}$, entretanto, o entendimento de um mecanismo apropriado de absorção de umidade ainda não foi devidamente apresentado. Desta forma, este trabalho tem como objetivo principal avaliar a influência do efeito higrotérmico nas propriedades viscoelásticas do compósito termoplástico PPS /fibras de carbono.

\section{Materiais e Métodos}

\subsection{Obtenção dos laminados e caracterização inicial}

O compósito PPS/fibra de carbono (PPS/FC) utilizado neste trabalho foi fornecido pela empresa holandesa Ten Cate Advanced Composites. O laminado fornecido apresentou uma espessura nominal de $1,86 \mathrm{~mm}$ e tecido de fibras de carbono com configuração $5 \mathrm{HS}$. 
Antes da realização do condicionamento higrotérmico e dos ensaios viscoelásticos os materiais foram caracterizados como recebidos, por ensaios de digestão ácida com o intuito de se avaliar o teor fibra/matriz. A digestão química da matriz é um método padronizado pela norma ASTM D 3171 que permite determinar o conteúdo de fibras de um laminado. Este consiste em um método simples para degradar a resina em um meio de digestão a quente sem atacar as fibras. O ácido sulfúrico concentrado é o meio de digestão selecionado para os laminados, especificado pelo Procedimento B da norma. Neste trabalho, este teste foi realizado em 6 amostras utilizando-se uma balança analítica de $0,0001 \mathrm{~g}$ de precisão e um digestor ácido da empresa Marconi. Após a digestão da matriz, os resíduos foram filtrados, lavados, secados e pesados e os conteúdos de fibra e resina foram determinados. A fração volumétrica de fibras e resina foi calculada a partir da massa de fibras obtidas após o ensaio de digestão ácida e dos valores teóricos das massas específicas destes constituintes.

\subsection{Condicionamento higrotérmico}

O condicionamento higrotérmico em câmara climática foi realizado de acordo com a norma ASTM D 5229/D 5229- 04. O controle de ganho de umidade foi realizado a partir de pesagens semanais da amostra condicionada, utilizando a Equação 1.

$$
\frac{\% \text { umidade }=(M u-M s)}{M s} .100 \%
$$

Onde: Mu: Massa da amostra úmida; Ms: Massa da amostra seca.

Para avaliar o efeito do condicionamento ambiental nos compósitos poliméricos estudados, cinco amostras de acompanhamento e três corpos de prova (cdps) para análise térmica de DMA foram submetidos a esse condicionamento. As amostras foram expostas à temperatura de $80^{\circ} \mathrm{C}$, onde o teor de umidade relativa selecionado foi de $90 \%$ por um período de oito semanas (tempo necessário para se atingir a saturação de umidade). Estes parâmetros foram programados em uma câmara de condicionamento higrotérmico Marconi modelo MA 835/UR disponível no Laboratório de Condicionamento Ambiental do Departamento de Materiais e Tecnologia (DMT), da UNESP, Campus de Guaratinguetá.

Antes de iniciar a climatização, todos os cdps e as amostras de controle foram secos em uma estufa a vácuo da marca Quimis modelo Q819V2 pertencente ao Laboratório de Cerâmica do DMT, por períodos médios de quarenta e oito horas na temperatura de $60{ }^{\circ} \mathrm{C}$. Após esse período de secagem as amostras de acompanhamento foram rapidamente removidas da estufa e pesadas em uma balança analítica Mettler Toledo, enquanto que os corpos de prova a serem disponibilizados para os ensaios dinâmico-mecânicos permaneceram na estufa. Em seguida, as amostras de acompanhamento e os cdps foram transferidos para a câmara de climatização e iniciado o condicionamento. A cada semana todas as amostras de acompanhamento eram removidas da câmara e pesadas para acompanhar o ganho de massa, até que a saturação fosse atingida. A partir dos dados de ganho de massa obtidos, foi possível construir um gráfico de ganho de massa médio em função do número de dias em que as amostras de acompanhamento e os cdps permaneceram dentro da câmara climática. Para avaliar se o sistema de absorção de umidade respeita ou não as leis de Fick, foram levantadas curvas de sorção em função do tempo representada pela Equação 2.

$$
M_{T} / M_{\infty}=k t^{n}
$$

Sendo $\mathrm{M}_{\mathrm{T}}$ relativo ao ganho de massa com relação ao tempo t, $\mathrm{M}_{\infty}$ relativo ao ganho de massa no equilíbrio e k e n são constante. Para sistemas fickianos, $\mathrm{n} \sim 1 / 2$, sistemas anômalos $1 / 2<\mathrm{n}<1$ ou $\mathrm{n}<1 / 2$ e se o comportamento segue o caso II, $n \geq 1^{[26]}$.

Sendo:

I. Difusão Fickiana em que a taxa de difusão é menor que a mobilidade dos segmentos de cadeia. Neste caso o equilíbrio de sorção é rapidamente estabilizado, sendo dependente do tempo somente até um certo limite;

II. No outro extremo em que a taxa de difusão e mobilidade são maiores quando comparados a processos de relaxação das cadeias. Neste caso, o processo de sorção é fortemente dependente da cinética de intumescimento da matriz polimérica;

III. Difusão anômala, que ocorre quando a mobilidade de permeação da água e relaxação dos segmentos das cadeias são comparáveis. Quando a difusão é analisada com os polímeros no estado vítreo ou em regiões próximas à temperatura de transição vítrea, em que os segmentos têm pouca mobilidade, este comportamento pode ser encontrado ${ }^{[26]}$.

A Figura 1 apresenta, de forma esquemática, uma amostra exposta à umidade.

Para uma amostra de espessura "b", exposta em um ambiente úmido, como apresentado na Figura 1, o coeficiente da difusão D pode ser obtido a partir da Equação 3:

$$
\frac{M_{T}}{M \infty}=\frac{4}{b}\left(\frac{D}{\pi}\right)^{0,5} t^{0,5}
$$

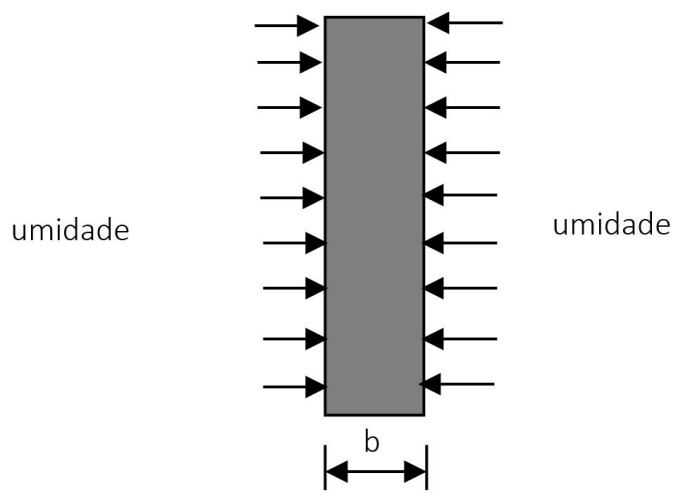

Figura 1. Representação esquemática da difusão de água em uma dimensão. 


\subsection{Análise dinâmico mecânica (DMA)}

Com o intuito de se avaliar o efeito higrotérmico nas propriedades viscoeláticas e na transição vítrea dos laminados de PPS/FC foram realizados ensaios de DMA. Neste trabalho foi utilizado um DMA da TA Instruments, modelo 2980, disponível na Escola de Engenharia Química da USP, Campus de Lorena EEL-USP, operando em modo de varredura por flexão, faixa de temperatura de 20 até $250^{\circ} \mathrm{C}$, com razão de aquecimento de $3^{\circ} \mathrm{C} / \mathrm{min}$ e amplitude de oscilação de $10 \mu \mathrm{m}$. Os ensaios de DMA foram realizados com o intuito de se avaliar o efeito higrotérmico nas propriedades viscoelásticas e na temperatura de transição vítrea dos laminados PPS/FC. Utilizando-se desta técnica, três amostras para cada situação foram analisadas.

\section{Resultados e Discussão}

\subsection{Digestão ácida da matriz polimérica}

A Tabela 1 apresenta a média dos resultados do teor de fibras de carbono nos laminados avaliados neste estudo, tendo sido avaliadas 5 amostras para cada caso. Os valores encontrados quanto ao teor volumétrico de fibras de carbono foram próximos aos valores fornecidos pela empresa fornecedora destes laminados termoplásticos, sendo estes de aproximadamente $60 \%$.

\subsection{Condicionamento higrotérmico}

A Figura 2 apresenta os valores de ganho de massa do laminado estudado em função do tempo de exposição em câmara climática. A análise dos dados apresentados indica que a concentração de umidade nas amostras de controle dos laminados dos compósitos PPS/FC, aumenta linearmente com o tempo na primeira semana de exposição. Nesta etapa, as amostras absorvem água muito rapidamente, alcançando um estado conhecido como pseudo-equilíbrio, mantendo a quantidade de água praticamente a mesma depois de um determinado período de tempo, sugerindo, em princípio, um comportamento Fickiano. A água permanece no compósito como água livre e tende, com o tempo, a penetrar na matriz polimérica pelo gradiente de concentração. Com a contínua exposição, o processo de absorção de umidade se torna mais lento e muitos autores atribuem a esse período, o início do processo de relaxação da cadeia polimérica e o preenchimento higrotérmico dos vazios existentes ${ }^{[12-14,23]}$.

Associado ao processo de sorção de umidade da matriz polimérica, outros mecanismos devem ser levados em consideração tais como a capilaridade e o transporte através de microtrincas e vazios. O mecanismo de capilaridade envolve o fluxo de água ao longo da interface/matriz seguido pela difusão através da interface até a matriz polimérica. $\mathrm{O}$ transporte de umidade por meio de microtrincas envolve tanto o fluxo como o armazenamento de água nos vazios e em outras formas de microdefeitos. Como o fluxo de água é proporcional ao gradiente de concentração, amostras expostas a ambientes com maiores teores de umidade podem apresentar maiores valores de absorção de umidade.

Dependendo do caso, esta difusão pode ainda gerar a entrada de umidade para regiões de volume livre, muitas vezes, resultando em um processo de inchamento da matriz polimérica.

Quando comparados com matrizes termorrígidas tais como a matriz epoxídica, pode ser constatado que mesmo utilizando elevados valores de temperatura e umidade, os laminados de PPS absorvem um baixo conteúdo de umidade. ${ }^{[10]}$ Este comportamento ocorre devido a diferenças entre a polaridade de matrizes epoxídicas e o PPS; pois quanto maior for a polaridade da matriz polimérica, maior será sua capacidade de absorção de água. Matriz de PPS apresenta um caráter menos polar levando, consequentemente, a uma menor absorção de umidade ${ }^{[10]}$.

As Figuras 3 e 4 apresentam os gráficos obtidos utilizando as Equações 1 (Figura 3) e 3 (Figura 4) e pontos a partir dos quais se fez regressão linear para a obtenção dos valores de $\mathrm{n}, \mathrm{k}$ e D para os laminados estudados. A partir dos resultados destas figuras, foi obtida a Tabela 2.

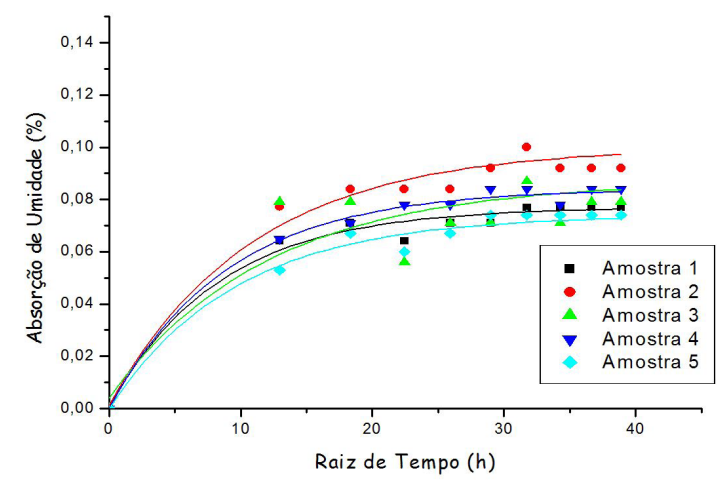

Figura 2. Resultados do ganho de massa em câmara de climatização.

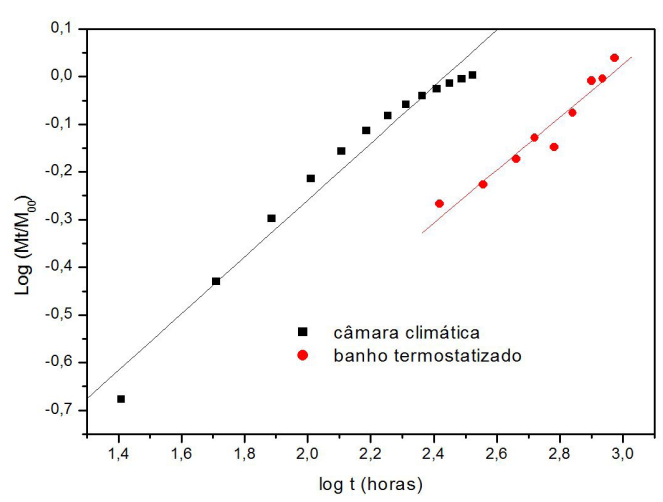

Figura 3. Avaliação dos parâmetros Fickianos para o compósito PPS/FC após ser submetido ao condicionamento ambiental.

Tabela 1. Valores dos teores de fibras de carbono nos laminados processados.

\begin{tabular}{ccc}
\hline \multicolumn{2}{c}{ Massa específica $\left(\mathbf{g} / \mathbf{c m}^{3}\right)$} & $\begin{array}{c}\text { Teor volumétrico } \\
\text { (\%) de fibra }\end{array}$ \\
\cline { 1 - 2 } PPS & Fibra & $61 \pm 6$ \\
\hline 1,43 & 1,91 & 6 \\
\hline
\end{tabular}

Tabela 2. Coeficiente de difusão e valores de n e k para os laminados em estudo.

\begin{tabular}{cccc}
\hline Laminado & $\mathbf{N}$ & $\mathbf{k}\left(\mathbf{g} / \mathbf{g} \cdot \mathbf{h}^{2}\right)$ & $\mathbf{D} \times \mathbf{~ 1 0}^{7}\left(\mathbf{c m}^{2} / \mathbf{m i n}\right)$ \\
\hline PPS/FC & 0,59 & 1,45 & 1,28 \\
\hline
\end{tabular}




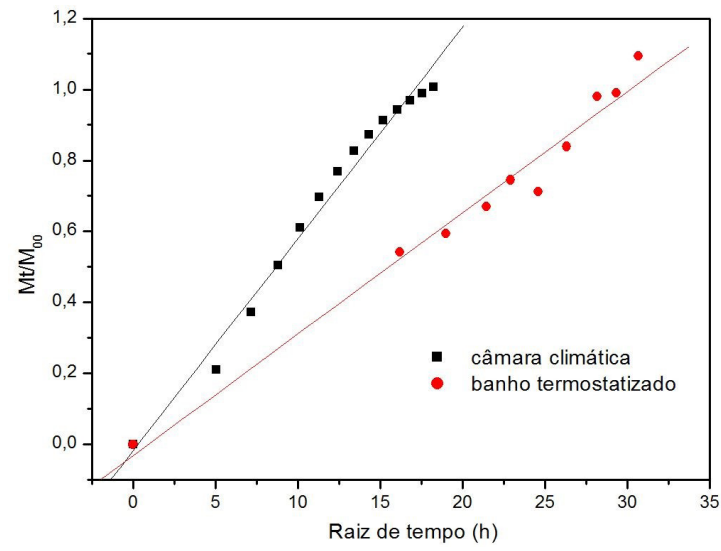

Figura 4. Avaliação da Difusividade para o compósito PPS/FC após ser submetido ao condicionamento ambiental.

Comparando-se os valores experimentais encontrados para o coeficiente de difusividade de laminados PPS/FC com os disponíveis em literatura para os compósitos de resina epoxídica/fibras de carbono, foi observado que estes laminados termoplásticos apresentam valores na ordem de três vezes menor (os valores para resina epoxídica variam de $5 \times 10^{-7}$ a $\left.35 \times 10^{-7} \mathrm{~cm}^{2} / \mathrm{min}\right)^{[27,28]}$. Estes dados, não só confirmam que os laminados termoplásticos obtidos a partir de matriz PPS absorvem menos umidade que os tradicionais laminados p processados com resina epoxídica, mas também validam os métodos de análise utilizados neste trabalho.

\subsection{Análise dinâmico mecânica}

De acordo com a literatura, a avaliação da temperatura de transição vítrea por técnicas diferentes do DMA é muito difícil $^{[26]}$. O fato da sensibilidade obtida pela técnica de DMA ser por volta de três ordens de grandeza superior à de outras técnicas de análises térmicas (tais como DSC, TM, etc) torna possível sua utilização para a determinação de transições físico-químicas fracas, normalmente imperceptíveis por outras técnicas. Por esta razão para este estudo foi escolhida a técnica de DMA para a avaliação de transições nos compósitos PPS/fibras de carbono ${ }^{[26]}$.

As Figuras $5 \mathrm{a}$ e b apresentam curvas representativas de DMA das amostras de PPS/FC, antes e após a climatização em câmara higrotérmica. Como pode ser observado, os valores do módulo de perda (E") apresentaram um aumento gradativo até a região de transição vítrea para todas as amostras analisadas, mostrando a necessidade de uma maior quantidade de energia para permitir que as cadeias poliméricas da fase amorfa adquiram maior mobilidade. Para todas as amostras foi constatado que os módulos de perda (E") apresentam picos máximos em temperaturas um pouco menores do que os picos máximos atribuídos para o atrito interno ( $\tan \delta=E$ '/E'). O máximo de dissipação de calor por unidade de deformação ocorre na temperatura em que E" é máximo. Esta temperatura, a $1 \mathrm{~Hz}$ de frequência, está mais próxima ao valor de temperatura de transição vítrea determinada por outros métodos, como DSC.

Desta maneira, pode-se utilizar o módulo de perda E" para se determinar a temperatura e/ou a região de transição vítrea dos materiais poliméricos. A Tabela 3 apresenta os valores de transição vítrea obtidos a partir de 3 amostras para cada

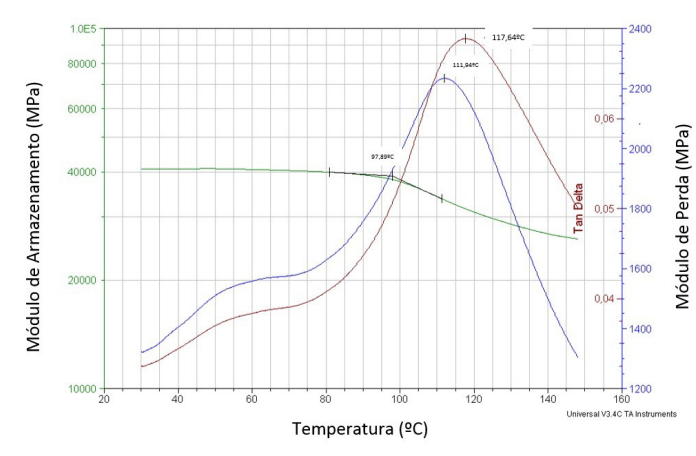

(a)

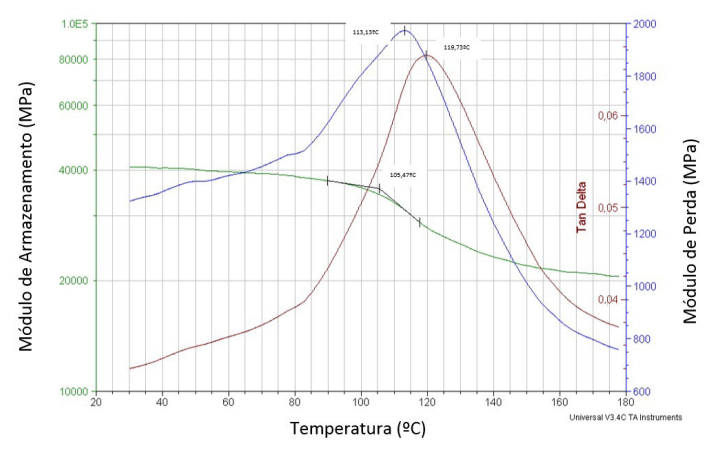

(b)

Figura 5. Curvas de DMA dos laminados PPS/FC: (a) condição não climatizada; (b) após condicionamento em câmara climática.

Tabela 3. Avaliação viscoelástica dos laminados de PPS/C.

\begin{tabular}{ccc}
\hline Propriedade & $\begin{array}{c}\text { PPS/FC } \\
\text { (antes do } \\
\text { condicionamento) }\end{array}$ & $\begin{array}{c}\text { PPS/FC } \\
\text { (após o } \\
\text { condicionamento) }\end{array}$ \\
\hline E" a $40^{\circ} \mathrm{C}(\mathrm{GPa})$ & $1,55 \pm 0,05$ & $1,50 \pm 0,10$ \\
E" na $\mathrm{T}_{\mathrm{g}}(\mathrm{GPa})$ & $2,35 \pm 0,15$ & $1,90 \pm 0,20$ \\
$\operatorname{Tan} \delta$ & $0,059 \pm 0,003$ & $0,065 \pm 0,002$ \\
$\mathrm{~T}_{\mathrm{g}}\left({ }^{\circ} \mathrm{C}\right)$ & $113,5 \pm 1,5$ & $113,0 \pm 0,5$ \\
\hline
\end{tabular}

situação apresentada na Figura 5 (gráficos representativos de todas as análises efetuadas). A partir desta Tabela e da Figura 5 pode ser observado que o valor médio da temperatura de transição vítrea nas amostras de $\mathrm{PPS} / \mathrm{FC}$ foi de $113,5^{\circ} \mathrm{C}$ (obtido no máximo de E"), respectivamente, sendo estas $30 \%$ superiores aos valores encontrados em literatura ${ }^{[10]}$.

Este fato sugere a presença de agentes tenacificantes ou outros constituintes presentes na formulação da matriz polimérica, não informados pelo fornecedor das amostras de compósitos. Este aumento dos valores da temperatura de transição vítrea, associados às variações dos valores dos módulos de perda e armazenamento no início da análise de DMA, quando comparadas às amostras climatizadas e não climatizadas, também sugere a presença de uma possível reticulação da matriz polimérica, induzida pela temperatura e umidade.

Ainda, de acordo com os dados apresentados na Figura 4, não foram observadas transições secundárias para a faixa de temperatura considerada neste trabalho. 


\section{Conclusões}

Os resultados obtidos a partir dos ensaios de digestão ácida estão de acordo com as especificações do fabricante quanto ao teor volumétrico de fibras. A partir dos resultados obtidos com relação às análises dinâmico-mecânicas, foi constatado que o efeito de absorção de umidade para as amostras de PPS/FC não afetou significativamente a integridade do laminado, entretanto, após o condicionamento em câmara climática, foi observado que tanto a temperatura de transição vítrea quanto a energia de dissipação aumentaram. Estes dados revelam que as moléculas de água provavelmente ocasionam um efeito de reticulação, concorrendo com o efeito plastificante sobre o sistema, provocando um aumento da $\mathrm{T}_{\mathrm{g}}$, proporcionando um "sistema modificado" $\mathrm{e}$, consequentemente, o aumento da sua temperatura de serviço.

\section{Agradecimentos}

Os autores gostariam de agradecer ao CNPq, CAPEs e FAPESP pelo auxílio financeiro dado para a realização deste trabalho de pesquisa.

\section{Referências}

1. Rezende, M. C., Nohara, E. L., Kawamoto, A. M., \& Nohara, L. B. (2007). Otimização da interface/interfase de compósitos termoplásticos de fibra de carbono/PPS pelo uso do Poli(ácido âmico) do tipo BTDA/DDS. Polímeros. Ciência e Tecnologia, 17(3), 180-187. http://dx.doi.org/10.1590/S010414282007000300005 .

2. Arici, A., Sinmazçelik, T., Çapan, L., Yilgor, I., \& Yilgor, E. (2005). Influence of annealing on the performance of short glass fiber-reinforced polyphenylene sulfide (PPS) composites. Journal of Composite Materials, 39(1), 21-33. http://dx.doi. org/10.1177/0021998305046432.

3. Gatti, C. A., Cardoso, F. L. A., Gualberto, A. R. M., Paz, J. H. H. A., Mota, L. P., Simêncio, E. C. A., Almeida, E. G. R., \& Tarpani, J. R. (2009). Inspeção termográfica de danos por impacto em laminados de matriz polimérica reforçados por fibras de carbono. Polimeros: Ciência e Tecnologia, 19(4), 318328. http://dx.doi.org/10.1590/S0104-14282009000400012.

4. Costa, M. L., Botelho, E. C., Paiva, J. M. F., \& Rezende, M. C. (2005). Characterization of cure of carbon/epoxy prepreg used in aerospace field. Materials Research, 8(3), 317-322. http://dx.doi.org/10.1590/S1516-14392005000300016.

5. Eslami, S., Honarbakhsh-Raouf, A., \& Eslami, S. (2015). Effects of moisture absorption on degradation of E-glass fiber reinforced Vinyl Ester composite pipes and modelling of transiente moisture diffusion using finite elemento analysis. Corrosion Science, 90, 168-175. http://dx.doi.org/10.1016/j. corsci.2014.10.009.

6. Neto, F. L., \& Pardini, L. C. (2006). Compósitos estruturais: ciência e tecnologia. São Paulo: Edgar Blücher.

7. Deroiné, M., Le Duigou, A., Corre, Y.-M., Le Gac, P.-Y., Davies, P., César, G., \& Bruzaud, S. (2014). Accelerated ageing of polylactide in aqueous environments: comparative study between distilled water and seawater. Polymer Degradation \& Stability, 108, 319-329. http://dx.doi.org/10.1016/j. polymdegradstab.2014.01.020.

8. Rezende, M. C., Costa, M. L., Botelho, E. C., \& Mazur, R. L. (2008). Avaliações térmica e reológica da matriz termoplástica PEKK utilizada em compósitos aeronáuticos. Polímeros: Ciência e Tecnologia, 18(3), 237-243. http://dx.doi.org/10.1590/ S0104-14282008000300009.
9. Garcia-Gonzalez, D., Rusinek, A., Jankowiak, T., \& Arias, A. (2015). Mechanical impact behavior of polyether-ether-ketone (PEEK). Composite Structures, 124, 88-99. http://dx.doi. org/10.1016/j.compstruct.2014.12.061.

10. Blond, D., Vieille, B., Gomina, M., \& Taleb, L. (2014). Correlation between physical properties, microstructure and thermo-mechanical behavior of PPS-based composites processed by stamping. Journal of Reinforced Plastics and Composites, 38, 1-13. http://dx.doi.org/10.1177/0731684414541846.

11. Bismarck, A., Hofmeier, M., \& Dörner, G. (2007). Effect of hot water immersion on the performance of carbon reinforced unidirectional poly(ether ether ketone) (PEEK) composites: stress rupture under end-loaded bending. Composites. Part A, Applied Science and Manufacturing, 38(2), 407-426. http:// dx.doi.org/10.1016/j.compositesa.2006.03.004.

12. Cândido, G. M. (2001). Influência do condicionamento ambiental na resistência à delaminação de borda livre em compósitos avançados (Tese de doutorado). InstitutoTecnológico de Aeronáutica, São José dos Campos.

13. Rezende, M. C., Cândido, G. M., \& Mayer, S. (2003). Influência do condicionamento ambiental na resistência à tração de compósitos de carbono/epóxi reparados. Polímeros: Ciência e Tecnologia, 13(3), 147-153. http://dx.doi.org/10.1590/S010414282003000300004.

14. Rezende, C. M., Costa, L. M., \& Cunha, J. A. P. (2006). Influência de diferentes condições higrotérmicas na resistência à tração de compósitos de fibra de carbono/epóxi modificada. Polímeros: Ciência e Tecnologia, 16(3), 193-201. http://dx.doi. org/10.1590/S0104-14282006000300008.

15. Botelho, E. C., Costa, M. L., Pardini, L. C., \& Rezende, M. C. (2005). Processing and hygrothermal effects on viscoelastic behavior of glass fiber/epoxy composites. Journal of Materials Science, 40(14), 3615-3623. http://dx.doi.org/10.1007/s10853005-0760-2.

16. Botelho, E. C., Pardini, L. C., \& Rezende, M. C. (2006). Hygrothermal effects on the shear properties of carbon fiber/ epoxy composites. Journal of Materials Science, 41(21), 7111-7118. http://dx.doi.org/10.1007/s10853-006-0933-7.

17. Botelho, E. C., Pardini, L. C., \& Rezende, M. C. (2007). Evaluation of hygrothermal effects on the shear properties of Carall composites. Materials Science and Engineering A, 452453, 292-301. http://dx.doi.org/10.1016/j.msea.2006.10.127.

18. Wang, Y., \& Hahn, T. H. (2007). AFM characterization of the interfacial properties of carbon fiber reinforced polymer composites subjected to hygrothermal treatments. Composites Science and Technology, 67(1), 92-101. http://dx.doi.org/10.1016/j. compscitech.2006.03.030.

19. Xiang, Z. D., \& Jones, F. R. (1997). Thermal-spike-enhanced moisture absorption by polymer-matrix carbon-fibre composites. Composites Science and Technology, 57(4), 451-461. http:// dx.doi.org/10.1016/S0266-3538(96)00168-6.

20. Yann, R., Damien, D., Rachid, E. G., \& Patrick, F. (2006). Anisotropy of hygrothermal damage in fiber/polymer composites: Effective elasticity measures and estimates. Mechanics of Materials, 38(12), 1143-1158. http://dx.doi.org/10.1016/j. mechmat.2006.02.001.

21. Zenasni, R., Bachir, A. S., Viña, I., Argüelles, A., \& Viña, J. (2006). Effect of hygrothermomechanical aging on the interlaminar fracture behavior of woven fabric fiber/PEI composite materials. Journal of Thermoplastic Composite Materials, 19(4), 385-398. http://dx.doi.org/10.1177/0892705706059743.

22. Chawla, K. K. (1998). Composite materials: science and engineering. New York: Springer. http://dx.doi.org/10.1007/9781-4757-2966-5.

23. Kawai, M., Morishita, M., Tomura, S., \& Takumida, K. (1998). Inelastic behavior and strength of fiber-metal hybrid composite: 
Glare. International Journal of Mechanical Sciences, 40(2-3), 183-198. http://dx.doi.org/10.1016/S0020-7403(97)00048-9.

24. Boukhoulda, B. F., Adda-Bedia, E., \& Madani, K. (2006). The effect of fiber orientation angle in composite materials on moisture absorption and material degradation after hygrothermal ageing. Composite Structures, 74(4), 406-418. http://dx.doi. org/10.1016/j.compstruct.2005.04.032.

25. Baker, A., Dutton, S., \& Kelly, D. (2004). Composite materials for aircraft structures. Virginia: American Institute of Aeronautics and Astronautics.

26. Faria, M. C. M. (2008). Avaliação do efeito higrotérmico nas propriedades mecânicas de compósitos de PPS/fibras contínuas (Dissertação de mestrado). Universidade Estadual Paulista, Guaratinguetá.
27. Batista, N. L. (2012). Influência das intempéries nas propriedades térmicas e mecânicas de compósitos PEI/fibras de carbono (Dissertação de mestrado). Instituto Tecnológico de Aeronáutica, São José dos Campos.

28. Costa, M. L., Rezende, M. C., Botelho, E. C., \& Costa, G. G. (2008). Avaliação do ciclo térmico de conformação por compressão de peças em poli(sulfeto de fenileno) reforçado com fibras contínuas de carbono. Polímeros: Ciências e Tecnologia, 18(1), 81-86. http://dx.doi.org/10.1590/S010414282008000100016 .

Enviado: Jul. 07, 2015

Revisado: Fev. 04, 2016

Aceito: Mar. 30, 2016 